\title{
Correspondence
}

\section{SIMULATED AND REAL ECT}

DEAR SIR,

In modern studies of the efficiency of ECT lack of careful recording of the seizure activity is not acceptable. It is not satisfactory only to state that a bilateral modified convulsion is evoked (Lambourn and Gill, Journal, December 1978, 132, 514-19). This may imply everything from a maximal generalized seizure to a submaximal seizure influenced by drugs with anti-epileptic action, reflecting variations from high to negligible anti-depressive efficiency. Lacking EEG-recording of the seizure activity in the cited paper, a low anti-depressive efficiency may be suspected in view of the facts that benzodiazepines had been given and the outcome after six treatments was unimpressive.

The paper gives a new illustration that the best way to 'prove' the inefficiency of a treatment is to give it in inadequate dose to small patient series using a measuring instrument of unaccounted reliability.

More is required before 40 years' of clinical experience and scientific evidence is thrown away and the essential role of the seizure activity in ECT is questioned.

University of Göteborg,

Jan-Otto Otrosson

Department of Psychiatry,

Sahlgrenska Sjukhuset,

41345 Göteborg, Sweden

DeAR SiR,

I should like to make four comments on the paper by Lambourn and Gill (Joumal, December 1978, 133, 514-19). Although one means of assessing outcome was to be 'days in hospital' there is no reference to this in the results or discussion sections. As a possible explanation for the alleged similar outcome for both groups 'the possibility was examined that a sub-group of patients did well but their responses were masked by our presentation of mean results; the distribution of good responses were similar between the groups, and no clinical features distinguished them'. However, the authors did not consider whether or not a sub-group of the patients receiving simulated ECT (S) may have fared worse than those receiving real treatment $(\mathbf{R})$. If one examines the assessments after six treatments by 'Hamilton outcome improvement' it is seen that outcome is as follows: $+++6 R$ and $6 S ;++5 R$ and $5 S ;+4 R$ and $1 S ; 01 R$ and $4 S$. Thus when poor outcome $(+$ and 0$)$ is considered there is no doubt that those receiving $S$ are overrepresented. Examination of 'referring doctors' global assessment of outcome' also reveals a preponderance of poor responders in the $\mathrm{S}$ group.

My final two comments concern the administration of the treatments thrice weekly to a total of 6 . It seems possible that, whatever the mode of action of ECT, longer intervals between applications over a longer period are required for maximum benefit as in the more usual practice of giving treatment twice weekly. Also, more than six treatments may be required. There is suggestive evidence from the study itself in support of one or both of these points. It is only suggestive since drugs were also administered, 6 patients were lost to follow up and patient groups were small. Of patients receiving further ECT over and above the initial 6, 4 improved in the $R$ group and 5 in the $S$ with no relapses in either. Of those receiving no further ECT 1 improved and 2 relapsed in the $\mathbf{R}$ group, the figures for the $S$ group being $\mathbf{2}$ and 2.

Thus, bearing in mind the small numbers and other caveats, this study could well be interpreted as supporting the view that real ECT is more effective than the simulated version.

Gartnavel Royal Hospital, 1055 Great Western Road, Glasgow G12 OXH

\section{OCGIPITAL ALPHA ACTIVITY AND PERSONALITY}

DeAR Sir,

Kondo, Bean, Travis and Knott (Journal, April $1978,132,378-80$ ) draw three conclusions from their experiment on EEG alpha activity and personality, namely: 1. 'the data do not support Eysenck's hypothesis concerning the relationship of cortical activity to personality types'; 2 . 'both scales of the EPI can be reliably related to occipital alpha'; 